\title{
Reactions of Trimethylsilylated Silicate Anions in Acidic Medium. Part 1. Effect of Amberlyst 15 Cation-exchange Resin on the Products of Tri- methylsilylation of Hexameric Ring Silicates
}

By Gabriella Garzó, * Anna Vargha, and Tamás Székely, Hungarian Academy of Sciences, Research Labora tory for Inorganic Chemistry, Budapest, Hungary

Dagobert Hoebbel, Academy of Sciences of the GDR, Central Institute for Inorganic Chemistry, BerlinAdlershof, German Democratic Republic

\begin{abstract}
Chemical and structural transformations induced by proton-donor catalysts (Amberlyst 15 cation-exchange resin and trichloroacetic acid) have been studied using the silicate derivatives $\mathrm{Si}_{6} \mathrm{O}_{15}\left(\mathrm{SiMe}_{3}\right)_{6}$ and $\mathrm{Si}_{6} \mathrm{O}_{18}\left(\mathrm{SiMe}_{3}\right)_{12}$ as model compounds. The experiments were performed in hexamethyldisiloxane solutions using a standard quality Amberlyst 15. Gas chromatography with a glass capillary column was used for detection of the composition changes in the reaction mixtures. Present and former results have permitted a probable structural presentation of the reaction pathways to be made. The transformations tend to produce structures containing only tetrameric siloxane rings when starting from cyclic and polycyclic silicate derivatives containing trimeric, (pentameric), or hexameric siloxane rings. Three different reaction types are suggested for the interpretation of the transformations. Amberlyst 15 was found to contribute significantly to side reactions during the Amberlyst treatment following the trimethylsilylation of silicates under the usual conditions employed.
\end{abstract}

TRIMETHYLSILYLATION of low-molecular-weight silicate systems has been widely used since 1964 when Lentz ${ }^{1}$ reported that trimethylsilyl esters of silicates were relatively volatile and fairly stable compounds, soluble in organic solvents. The technique of trimethylsilylation usually precedes gas chromatographic (g.c.) and mass spectrometric (m.s.) investigations, in order to volatilize the silicate species while preserving its original structure. However, when silicates of known structure are examined using any of the recommended trimethylsilylation techniques, it appears that most of the silica is lost and/or recovered as derivatives of anions other than those originally present in the parent compound. This indicates the occurrence of undesirable side reactions, which are, in the opinion of Götz and Masson, ${ }^{2}$ polymerization and depolymerization processes taking place before trimethylsilylation has been completed, and hydrolysis of the trimethylsilyl derivatives themselves.

Recently Calhoun and Masson ${ }^{3}$ and ourselves ${ }^{4}$ have stressed the importance of controlling the $\mathrm{pH}$ during the silylation reaction to avoid these disturbing side reactions.

Most investigators ${ }^{\mathbf{1 - 3 , 5}}$ agree that trimethylsilylation cannot be completed without after-treatment of the organic phase (hexamethyldisiloxane solution) of the reaction mixture with Amberlyst 15 cation-exchange resin for several hours. This after-treatment is supposed to promote the proton-trimethylsilyl exchange in some residual shielded $\mathrm{OH}$ groups in the silicate skeleton.

Calhoun and Masson ${ }^{3}$ have also found that, besides completing the trimethylsilylation, Amberlyst 15 may interconvert certain trimethylsilyl-silicate isomers and contribute to the occurrence of other side reactions. ${ }^{6}$

In an earlier paper ${ }^{4}$ we proposed a new trimethylsilylation method for aqueous silicic acid solutions, using bis(trimethylsilyl)acetamide as the silylating agent. In this method after-treatment with Amberlyst proved to be unnecessary. In another paper on tetraethylammonium silicates ${ }^{7}$ we reported that using the silylation method developed by Tamás et al. ${ }^{5}$ trimethylsilyl ester formation was again complete without Amberlyst treatment.

In this paper we demonstrate the contribution of strong acids (Amberlyst 15 and trichloroacetic acid) to the side reactions of the trimethylsilylation procedure. It is shown that these acids give rise to characteristic chemical transformations of the trimethylsilyl silicates themselves.

The chemical processes discussed here were examined in reaction mixtures containing hexamethyldisiloxane and Amberlyst or trichloroacetic acid. Crystalline

(a)

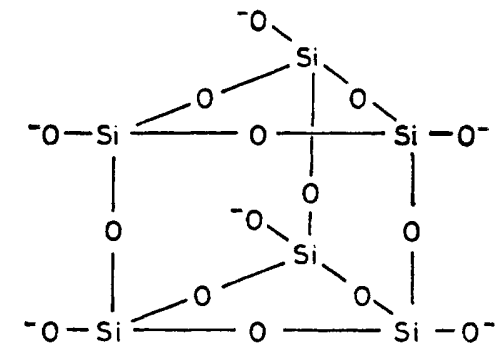

(b)

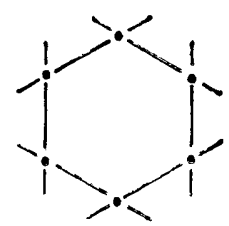

FIGURE 1 Structures of the model compounds

tetraethylammonium silicate and dioptase were chosen as the model systems. The anionic component of crystalline tetraethylammonium silicate is a double trimeric ring silicate ${ }^{7}$ and that of dioptase is a hexameric ring silicate. ${ }^{8}$ The original structure of tetraethylammonium silicate is shown in Figure $1(a)$ and that of dioptase in Figure $1(b) .{ }^{8}$ The dioptase structure is pictured in the simplified form used for all silicates and their trimethylsilyl derivatives throughout this paper. 
Making use of the abbreviations, $\mathrm{Si}\left(\mathrm{O}_{0.5}\right)_{4}=\mathrm{SiO}_{2}=\mathrm{Q}$ and $\mathrm{Me}_{3} \mathrm{SiO}_{\mathbf{0 . 5}}=\mathrm{L}$, the trimethylsilylated product from tetraethylammonium silicate can be designated as $\mathrm{Q}_{6} \mathrm{~L}_{6}$ and that from dioptase as $\mathrm{Q}_{6} \mathrm{~L}_{12}$.

\section{EXPERIMENTAL}

The preparation and trimethylsilylation of crystalline tetraethylammonium silicate have already been reported. ${ }^{7}$ The chromatogram of the silylation product showed one main peak with area $92 \%$; the elemental composition of the corresponding structure being $\mathrm{Si}_{6} \mathrm{O}_{15}\left(\mathrm{SiMe}_{3}\right)_{6}$.

Trimethylsilylation of Dioptase, $\mathrm{Cu}_{6} \mathrm{Si}_{6} \mathrm{O}_{18} \cdot 6 \mathrm{H}_{2} \mathrm{O}$.- A mixture of hexamethyldisiloxane $\left(5 \mathrm{~cm}^{3}\right)$, dimethylformamide $\left(10 \mathrm{~cm}^{3}\right)$, and $\mathrm{SiMe}_{3} \mathrm{Cl}\left(5 \mathrm{~cm}^{3}\right)$ was stirred for $15 \mathrm{~min}$ at room temperature. Dioptase powder $(39.7 \mathrm{mg})$ was then added and stirring was continued for a further $30 \mathrm{~min}$. The addition of $100 \mu \mathrm{l}$ of the internal standard solution $(6 \mathrm{mg}$ of $\mathrm{n}$-tetradecane in $1.3526 \mathrm{~g}$ of n-heptane) followed, and the mixture was washed with water $\left(9 \times 25 \mathrm{~cm}^{3}\right)$.

The yield of silylation measured by g.c. was $6 \%\left(\mathrm{SiO}_{2}\right)$ The chromatogram had one main peak, the retention value of which was characteristic of $12 \mathrm{SiMe}_{3}$ groups; area: $70 \%$; mass number of the corresponding compound: $1332 .{ }^{9}$

Standardization of the Amberlyst Resin.-Amberlyst 15 ion-exchange resin was supplied by Serva Feinbiochemica, Heidelberg. In order to standardize the ion exchanger, the contents of a freshly opened container were spread out on a plate in a thin layer and heated in an oven at $80^{\circ} \mathrm{C}$ for $4 \mathrm{~h}$. After cooling in a desiccator, the resin was stored in a dry place.

Characterization of the Amberlyst Resin.-Experience gained over the years has shown that different batches of Amberlyst 15 have different activities in the completion of silylation reactions. We have also learned that the contribution of Amberlyst 15 to proton-trimethylsilyl exchange is preceded by the cleavage of an $\equiv \mathrm{Si}-\mathrm{O}-\mathrm{Si} \equiv$ bond in hexamethyldisiloxane on addition of the protonated Amberlyst 15. It was therefore expected that $\equiv \mathrm{Si}-\mathrm{O}-\mathrm{Si} \equiv$ bonds in longer methylsiloxane chains would likewise be cleaved by Amberlyst 15.

For practical reasons, based on our experience, the rate of disappearance of the dodecamethylpentasiloxane peak in the chromatogram, in the presence of Amberlyst 15, was chosen as the characteristic feature of Amberlyst activity. For comparison, pure Amberlyst 15 from the same bottle without any pretreatment and after the standardization procedure was used to treat dodecamethylpentasiloxane.

Dodecamethylpentasiloxane $(1 \mathrm{mg})$, n-heptane $(500 \mu \mathrm{l})$, standard quality Amberlyst $15(20 \mathrm{mg})$, and a stock solution of the internal standard $(25 \mu \mathrm{l})$ were shaken at $20{ }^{\circ} \mathrm{C}$ for several days. From time to time, $1-\mu l$ aliquots were directly injected into the gas chromatograph.

Comparison of the curves in Figure 2 shows the effect of the standardization procedure. At the same time, the upper curve characterizes the standard quality Amberlyst 15 used throughout this work. Both curves reflect dodecamethylpentasiloxane consumption in the absence of hexamethyldisiloxane.

The heat treatment of Amberlyst 15 at $80{ }^{\circ} \mathrm{C}$ represents a drying process, which does not alter the chemical composition or the structure of the resin.10 As a result of standardization the nature of the catalysed reaction remains unchanged, but the activity of the catalyst is increased.

Amberlyst Treatment of $\mathrm{Si}_{6} \mathrm{O}_{15}\left(\mathrm{SiMe}_{3}\right)_{6}$ and $\mathrm{Si}_{6} \mathrm{O}_{18}\left(\mathrm{SiMe}_{3}\right)_{12}$.
-A solution of $\mathrm{Si}_{6} \mathrm{O}_{15}\left(\mathrm{SiMe}_{3}\right)_{6}\left(c a .1 .5 \mathrm{~cm}^{3}\right)\left(0.2 \mathrm{mg}\right.$ of $\mathrm{SiO}_{2}$ per $1 \mathrm{~cm}^{3}$ of hexamethyldisiloxane) was evaporated to dryness; hexamethyldisiloxane $(200 \mu \mathrm{l})$, n-heptane $(200$ $\mu 1)$, and Amberlyst $15(40 \mathrm{mg})$ were then added.

A solution of $\mathrm{Si}_{6} \mathrm{O}_{18}\left(\mathrm{SiMe}_{3}\right)_{12}\left(4 \mathrm{~cm}^{3}\right)\left(0.09 \mathrm{mg}\right.$ of $\mathrm{SiO}_{2}$ per $1 \mathrm{~cm}^{3}$ of hexamethyldisiloxane) was evaporated to dryness. Hexamethyldisiloxane $\left(1 \mathrm{~cm}^{3}\right)$, standard quality Amberlyst $15(500 \mathrm{mg})$, and a stock solution of the internal standard $(50 \mu l)$ were then added.

Both samples were shaken at $20{ }^{\circ} \mathrm{C}$ for several days.

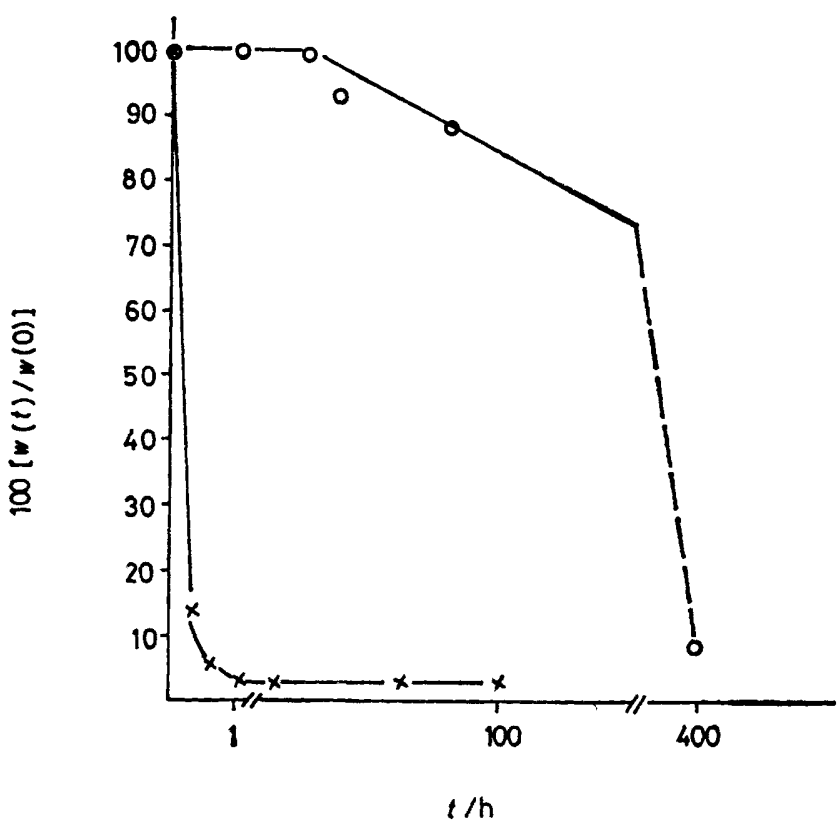

Figure 2 Characterization of Amberlyst. Recovery of the original weight, $w(0)$, of dodecamethylpentasiloxane according to the chromatograms after Amberlyst treatment for $t \mathrm{~h}$ : dodecamethylpentasiloxane $\left(1.3 \times 10^{-4} \mathrm{mmol}\right)$ per $1 \mathrm{mg}$ of Amberlyst in $n$-heptane without any treatment of the original ionexchanger $(O)$; and after drying the original ion-exchanger according to the procedure given in the text $(x)$

From time to time $0.5-4 \mu \mathrm{l}$ aliquots were directly injected into the gas chromatograph.

Treatment of $\mathrm{Si}_{6} \mathrm{O}_{15}\left(\mathrm{SiMe}_{3}\right)_{6}$ with Trichloroacetic Acid.--To $1 \mathrm{~cm}^{3}$ of a $\mathrm{Si}_{6} \mathrm{O}_{15}\left(\mathrm{SiMe}_{3}\right)_{6}$ solution $\left(0.9 \mathrm{mg} \mathrm{SiO}{ }_{2}\right.$ per $1 \mathrm{~cm}^{3}$ of hexamethyldisiloxane) was added trichloroacetic acid (170 $\mathrm{mg}$ ) to obtain a $1.0 \mathrm{~mol} \mathrm{dm}^{-3}$ solution of trichloroacetic acid. The sample was shaken and injected into the gas chromatograph as before.

Gas Chromatography.-A Hewlett-Packard model 5830/A device equipped with a flame-ionization detector and capillary inlet system model 18740/A was used. The column $(20 \mathrm{~m} \times 0.25 \mathrm{~mm}$ diameter $)$ was of Pyrex glass. The stationary phase was $O V-1$ (phase ratio $\beta \cong 600$ ) and the carrier gas was nitrogen. Operating conditions were: linear velocity, $30 \mathrm{~cm} \mathrm{~s}^{-1}$; injection-port temperature, $280{ }^{\circ} \mathrm{C}$; detector block temperature, $300{ }^{\circ} \mathrm{C}$. The temperature of the column was increased at $10^{\circ} \mathrm{min}^{-1}$ from an initial temperature of $115^{\circ} \mathrm{C}$ to a final temperature of $300{ }^{\circ} \mathrm{C}$. n-Tetradecane was used as internal standard. The percentage values in Figures $4-6$ denote the area percentages, corrected by the following individual flameionization detector response factors (= amount/area): n-tetradecane, $1 ; \mathrm{QL}_{4}, 2.5 ; Q_{n} \mathrm{~L}_{n+2}, 2.0 ; \mathrm{Q}_{n} \mathrm{~L}_{2 n}, 1.7 ; \mathrm{Q}_{n} \mathrm{~L}_{n}$, $3.3 ; \mathrm{Q}_{8} \mathrm{~L}_{10}, 1.3 ; \mathrm{Q}_{8} \mathrm{~L}_{8}, 1.5$. 


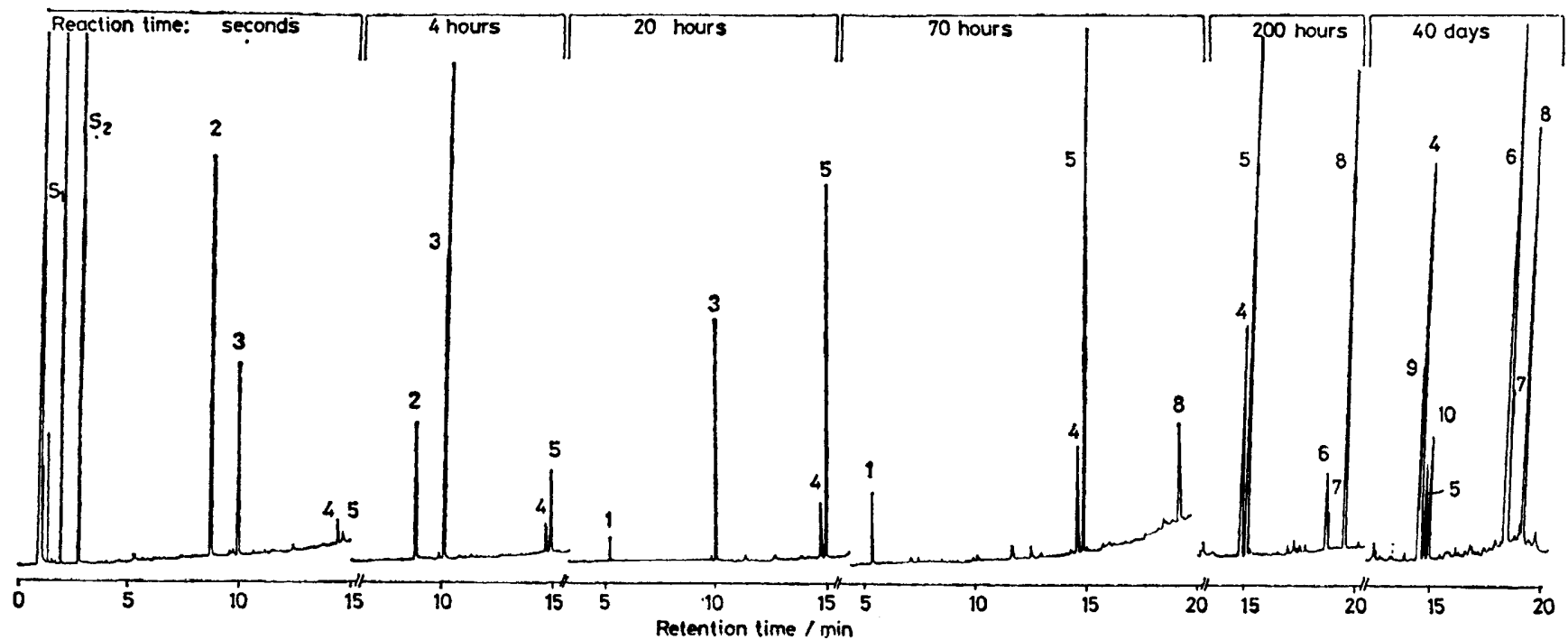

FIGURE 3 Chromatograms taken during Amberlyst treatment of $\mathrm{Q}_{\mathrm{g}} \mathrm{L}_{\mathrm{g}}$ : dodecamethylpentasiloxane internal standard peak (S): n-tetradecane internal standard peak $\left(\mathrm{S}_{2}\right) ; \mathrm{Q}_{2} \mathrm{~L}_{6}(1) ; \mathrm{Q}_{6} \mathrm{~L}_{6}(2) ; \mathrm{Q}_{8} \mathrm{~L}_{8}(3) ; \mathrm{Q}_{8} \mathrm{~L}_{10}$ (I) (4); $\mathrm{Q}_{8} \mathrm{~L}_{10}$ (II) (5); $\mathrm{Q}_{6} \mathrm{~L}_{12}$ (I) (6); ( $\mathrm{O}_{8} \mathrm{~L}_{12}(\mathrm{lia})$ (7); $Q_{6} L_{12}$ (II) (8); $Q_{6} L_{10}(9)$ and (10). Designations of $Q_{6} L_{12}$ (I) and $Q_{6} L_{12}$ (II) correspond to thosc in ref. 3 . For the g.c. conditions see the text

\section{RESULTS}

Chemical Transformations of $\mathrm{Q}_{6} \mathrm{~L}_{6}$.- - The processes occurring during Amberlyst treatment are qualitatively demonstrated by the series of chromatograms in Figure 3. Peak assignments were performed partly with the help of refer- ence materials $\left[Q_{2} L_{6}, Q_{6} L_{10}(\mathrm{I}), Q_{6} L_{10}(\mathrm{II}), 11\right.$ and $\left.Q_{6} L_{6}\right]$ and partly by g.c. $-m . s$. $\left[Q_{6} \mathrm{~L}_{8}, Q_{6} \mathrm{~L}_{12}(\mathrm{I}), Q_{6} \mathrm{~L}_{12}\right.$ (Ia), and $Q_{6} \mathrm{~L}_{12}$ (II) ${ }^{3,9}$. The composition of the silylation mixture remained unchanged over several months if Amberlyst was absent.

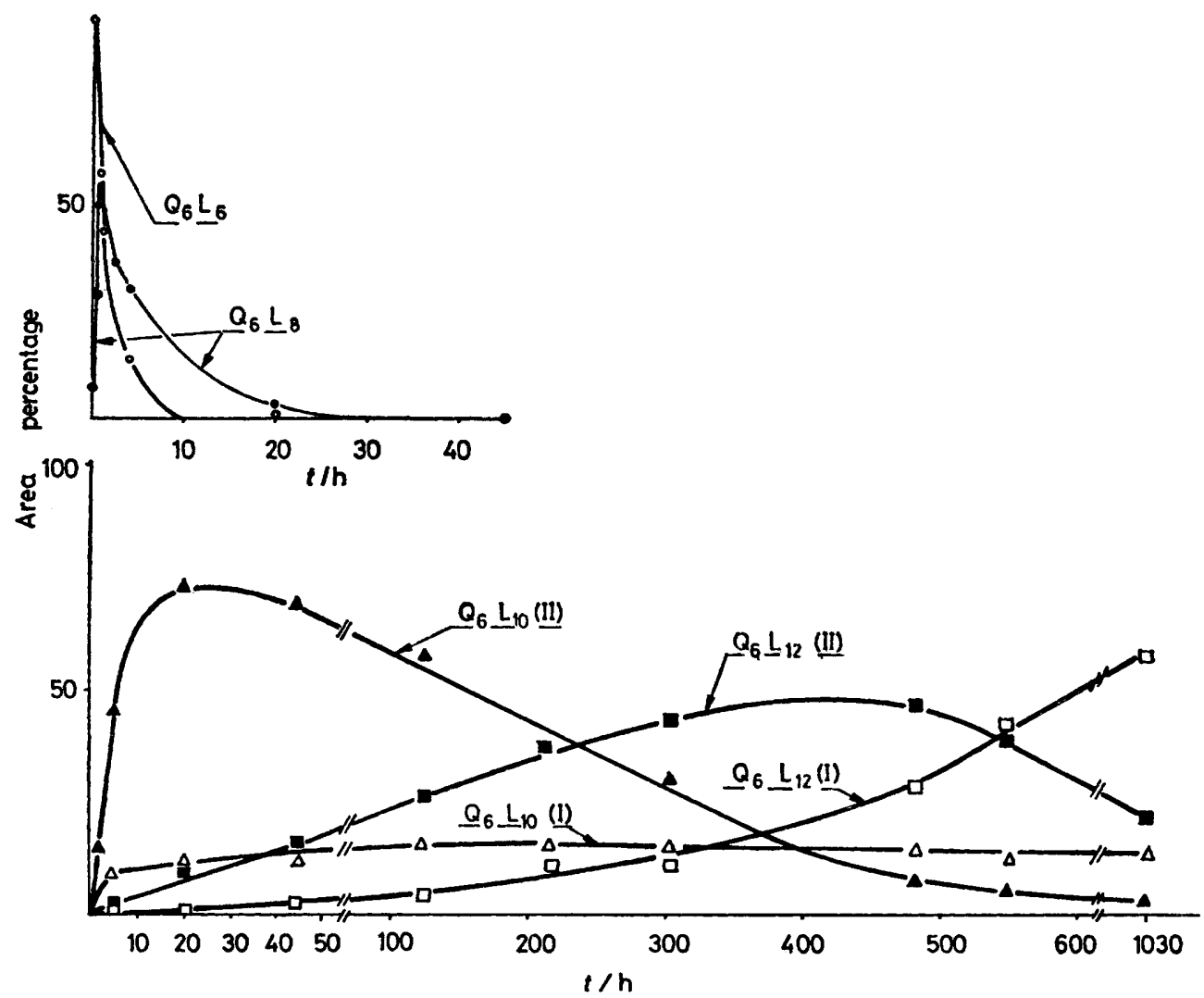

FIGURE 4 Concentration changes during the Amberlyst treatment of $\mathrm{Q}_{8} \mathrm{~L}_{8}$. Area percentages corrected by individual flame. ionization detector response factors. For conditions used for the Amberlyst treatment, see the Table 
The plots in Figure 4 show the concentration changes against time for the main reaction participants. Besides the corrected area percentage values, percentages of $\mathrm{SiO}_{2}$ were also calculated in several cases, by means of the internal standard added to the samples in known concentrations.4, ? No significant losses were found in the summarized $\mathrm{SiO}_{2}$ values during Amberlyst treatment.
$Q_{6} L_{6}$ similar to those observed above. The rate of the reactions is, however, rather slower upon treatment with trichloroacetic acid.

Chemical Transformations of $\mathrm{Q}_{6} \mathrm{~L}_{12}$. - The results of the Amberlyst treatment of the dioptase silylation product are illustrated in Figure 6. The sample contained one $Q_{6} \mathrm{~L}_{12}$ isomer as the main component. This was identified as

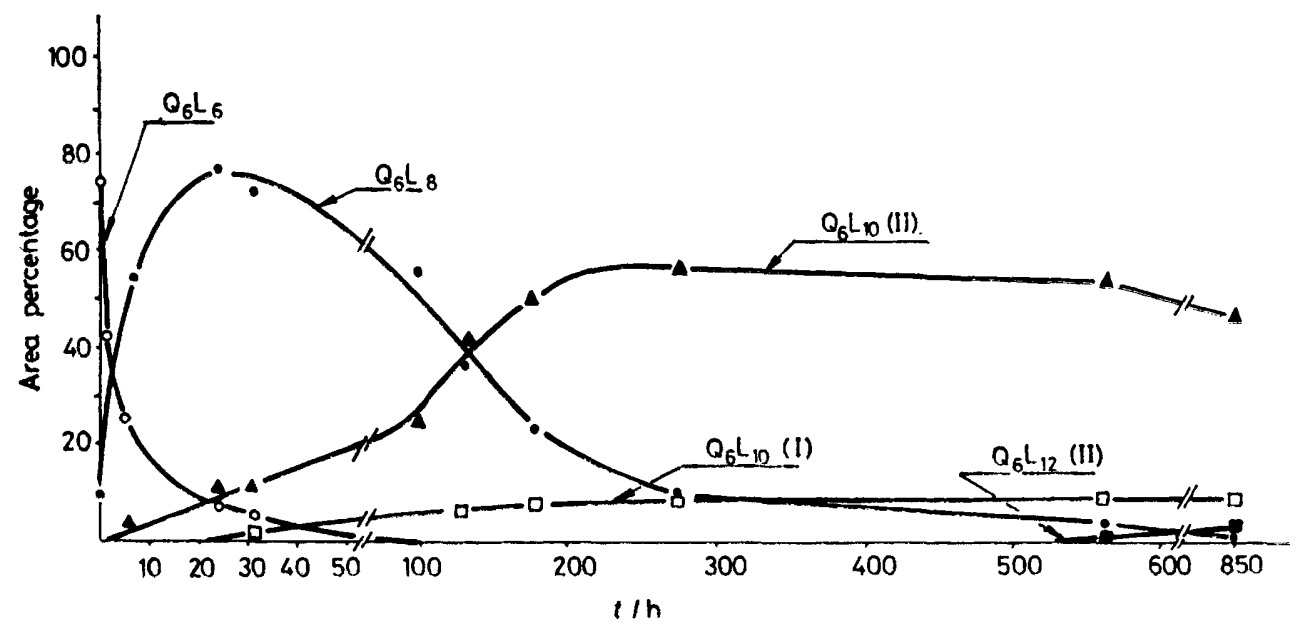

FIGURE 5 Concentration changes during the treatment of $Q_{6} \mathrm{~L}_{6}$ and $1 \mathrm{~mol} \mathrm{dm} \mathrm{m}^{-3}$ trichloroacetic acid. Areal percentages corrected by individual flame-ionization detector response factors

The diagrams show clearly the sequence and the clifferent rates of reactions (1)-(5). The formation, and

$Q_{6} L_{6} \quad \rightarrow Q_{B} L_{8} \quad$ very fast

$\mathrm{Q}_{6} L_{8} \quad \rightarrow Q_{6} L_{10}$ (II) slightly slower

$\mathrm{Q}_{6} \mathrm{~L}_{10}(\mathrm{II}) \rightarrow \mathrm{Q}_{6} \mathrm{~L}_{12}$ (II) substantially slower; medium rate

$\mathrm{Q}_{6} \mathrm{~L}_{12}(\mathrm{II}) \rightarrow \mathrm{Q}_{6} \mathrm{~L}_{12}$ (I) slow

$Q_{6} L_{10}(\mathrm{II}) \rightarrow Q_{6} \mathrm{~L}_{12}$ (I) slow

subsequent conversion, of $Q_{6} \mathrm{~L}_{10}(\mathrm{I})$ seems to reach a steady state at a rather low concentration.

In the diagram of Figure 5 the effect of trichloroacetic acid on the hexamethyldisiloxane solution of $Q_{6} L_{6}$ is demonstrated. The curves verify reactions starting from isomer (III) found already by Calloun and Masson ${ }^{3}$ in the Amberlyst treated silylation product of dioptase. Based on the diagrams, the consecutive reactions and the reaction rates are as given in equations $(6)-(10)$. As a result of

$Q_{6} \mathrm{~L}_{12}(\mathrm{III}) \rightarrow Q_{6} \mathrm{~L}_{12}$ (II) medium rate

$Q_{6} \mathrm{~L}_{12}$ (III) $\rightarrow Q_{6} \mathrm{~L}_{10}$ (I) slightly slower, medium rate (7)

$Q_{6} \mathrm{~L}_{12}(\mathrm{III}) \rightarrow Q_{6} \mathrm{~L}_{12}(\mathrm{I})$ slow

$\mathrm{Q}_{6} \mathrm{~L}_{12}(\mathrm{II}) \rightarrow \mathrm{Q}_{6} \mathrm{~L}_{12}(\mathrm{I})$ slow

$Q_{6} L_{10}(I) \rightarrow Q_{6} L_{12}(\mathrm{I})$ slow

experiments carried out under more or less similar conditions, Calhoun and Masson ${ }^{3}$ reported similar, though faster, changes in composition for the 20-170 h period of Amberlyst treatment; however, in contrast to our results, they found

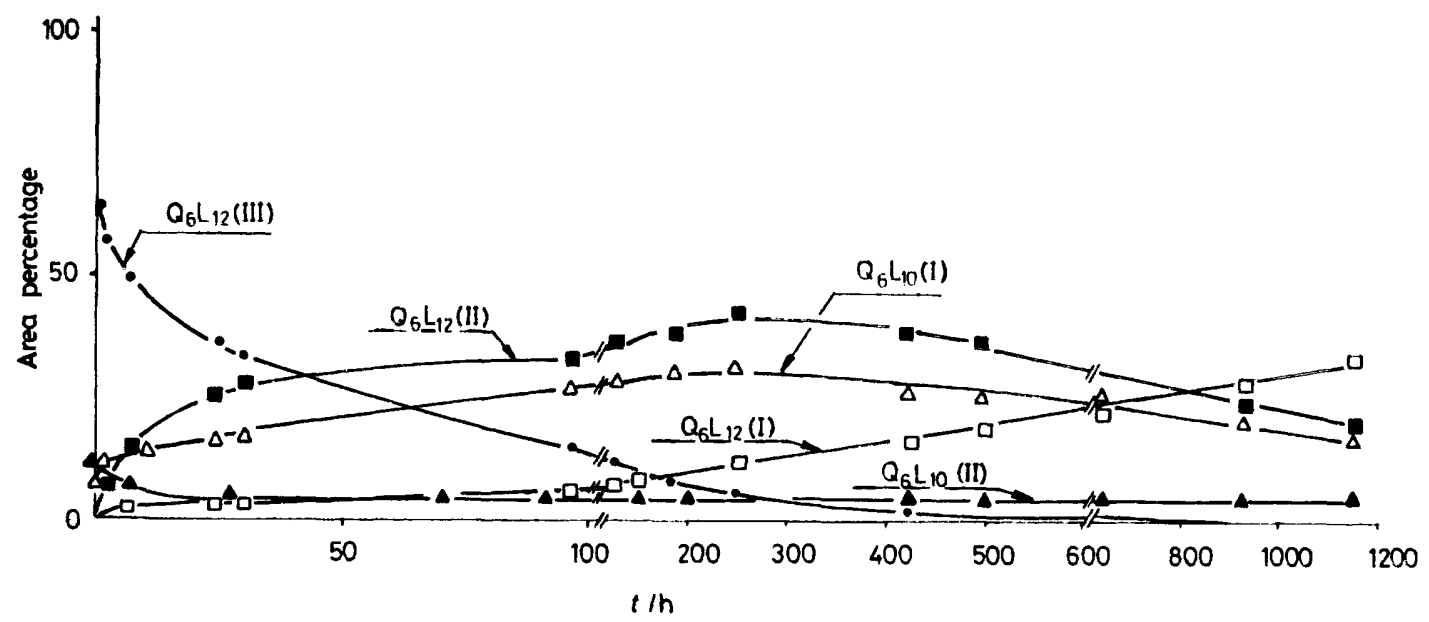

FIGURE 6 Concentration changes during the Amberlyst treatinent of $Q_{6} L_{12}$ (III). For conditions used for the Amberlyst treatment, see the Table. Area percentages corrected by individual flame-ionization detector response factors 
$Q_{6} L_{10}$ (I) to be the main component during the entire reaction period.

It is worth comparing the two sets of reactions for $Q_{6} L_{6}$ and $Q_{6} L_{12}$. As a result of several consecutive reactions $Q_{6} L_{6}$ is transformed into $Q_{6} L_{12}$ (I), but no $Q_{6} L_{12}$ (III) is formed. This fact was in good accordance with the lability, therefore the disappearance, of $Q_{6} \mathrm{~L}_{12}$ (III) from the dioptase silylation product. Starting either from $Q_{6} L_{6}$ or from $Q_{6} L_{12}$ (III), $\mathrm{Q}_{6} \mathrm{~L}_{12}$ (II) proved to be a common intermediate which is transformed in both cases into $Q_{6} \mathrm{~L}_{12}(\mathrm{I})$ [reactions (4) and (9)]. The role of $Q_{6} L_{10}$ (I) and (II) is quite different in the trimethylsilylating agent, e.g. Amberlyst + hexamethyldisiloxane.

Obviously the $\equiv \mathrm{Si}-\mathrm{O}-\mathrm{Si} \equiv$ bond is also split by trichloroacetic acid. Accordingly, Figure 5 demonstrates the occurrence of the same reactions in the same sequence. Owing to the low reaction rate, however, the type (iii) reaction could not be observed. This indicates that $(i)$ in proper conditions, similar to equilibrium (11), (11a) takes place as well (cf. Figure 7$) ;($ ii) the transitional product $\mathrm{Cl}_{3} \mathrm{CCOOSiMe}_{3}$ may be regarded as a ' silylating

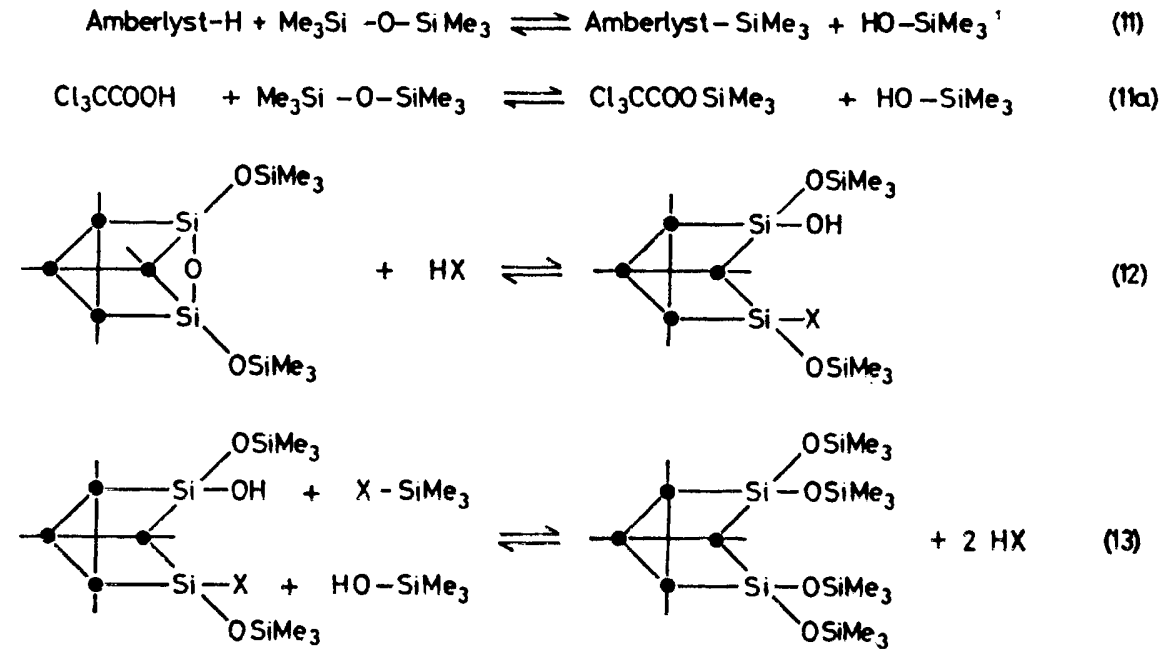

FIgURE 7 Mechanism of the type (i) and type (ii) reactions using the trinethylsilylated double trimeric ring as an example

two systems. In both reaction pathways the concentration of $Q_{6} L_{12}$ (I) increases monotonously and finally it becomes the main component of both reaction mixtures.

\section{DISCUSSION}

The Role of Amberlyst 15 and Trichloroacetic Acid.Reactions (1) to (10), listed above, represent three types of reactions. (i) In reactions (1), (2), (3), (5), and (10) a decrease in the condensation degree of the molecular structure via cleavage of one skeletal $\equiv \mathrm{Si}-\mathrm{O}-\mathrm{Si} \equiv$ bond followed by trimethylsilylation can be observed. (ii) In reaction (7) the condensation degree of the molecular structure increases, as the cleavage of two substituent $\equiv \mathrm{Si}-\mathrm{O}-\mathrm{Si} \equiv$ bonds is followed by the formation of a new skeletal $\equiv \mathrm{Si}-\mathrm{O}-\mathrm{Si} \equiv$ bond. (iii) In reactions (4), (6), (8), and (9) isomerization occurs via intramolecular rearrangement. Most probably the cleavage of one skeletal and one substituent $\equiv \mathrm{Si}-\mathrm{O}-\mathrm{Si} \equiv$ bond is followed by siloxane bond rearrangement and $\mathrm{OSiMe}_{3}$ group migration.

The role of Amberlyst 15 and trichloroacetic acid is obviously the participation in, or the catalysis of, these reactions. The mechanisms of type (i) and type (ii) reactions are similar to that of the well known equilibration processes of organosiloxanes, which can be catalyzed, among other agents, by proton-donor acids including the $\mathrm{H}^{+}$-form of cation-exchange resins. ${ }^{12}$ Type (i) reactions clearly postulate the presence of a agent ' for silicates similar to Amberlyst.SiMe ${ }_{3}$; and (iii) in order to explain the low reaction rate with trichloroacetic acid, three alternative or additive reasons may be considered: (a) Amberlyst 15 has an exceptionally high exchange capacity for $\mathrm{SiMe}_{3}$ groups and other $\equiv \mathrm{Si}-\mathrm{O}$ containing molecular fragments; $(b) \mathrm{Cl}_{3} \mathrm{CCOOSiMe}_{3}$ is a poorer 'silylating agent ' than Amberlyst.SiMe $\mathrm{S}_{3}$; $(c)$ the surface of the ion-exchange resin plays an important role in trimethylsilyl silicate transformations.

In conclusion, a rough approximation of the mechanism of type (i) and type (ii) reactions may be given. If $\mathrm{X}$ represents the Amberlyst matrix or the $\mathrm{Cl}_{3} \mathrm{CCOO}^{-}$ anion, then in addition to (11) and (11a), the reactions (12) and (13) (Figure 7) also occur. (The trimethylsilylated double trimeric ring serves only as an example in Figure 7.)

According to the mechanism of the type (i) reaction Amberlyst attacks concurrently hexamethyldisiloxane and a skeletal $\equiv \mathrm{Si}-\mathrm{O}-\mathrm{Si} \equiv$ bond of the trimethylsilylsilicate. The $\mathrm{H}^{+}$-form of the Amberlyst exerts a catalytic effect. However, regarding the huge excess of hexamethyldisiloxane compared to the silicates in our experiments, most of the catalyst is presumably consumed by means of reactions (11) and (11a). According to the mechanism of the opposite type (ii) reaction, Amberlyst attacks two substituent $\equiv \mathrm{Si}-\mathrm{O}-\mathrm{Si} \equiv$ bonds [reaction (13)]. This attack is followed by reaction (12), then by reaction $(11)$, each in reversed direction. This 
opposite pathway is found, however, only in case of the $Q_{6} \mathrm{~L}_{12} \longrightarrow \mathrm{Q}_{6} \mathrm{~L}_{10}$ transformation; hexamethyldisiloxane is produced instead of being consumed.

Structures and Stabilities of the Reaction Products.There have been ten components detected as the main initial, transitional, and end-products of the reactions starting from $Q_{6} \mathrm{~L}_{6}$ and $Q_{6} \mathrm{~L}_{12}(c f$. Figure 3$)$. The reaction pathway includes, however, only molecules with four different elementary compositions $\left(Q_{6} \mathrm{~L}_{6}, \mathrm{Q}_{6} \mathrm{~L}_{8}, \mathrm{Q}_{6} \mathrm{~L}_{10}\right.$, and $Q_{6} \mathrm{~L}_{12}$ ). Considering only the type (i) reaction and the fact that $Q_{6} L_{6}$ with a definite structure ${ }^{7}$ is the common starting material of the reaction products, the numbers of the possible geometric isomers for the remaining three compounds are: two for $Q_{6} L_{8}$, six for $Q_{6} L_{10}$, and nine for $Q_{6} L_{12}$ (cf. ref. 13 and Figure 8).

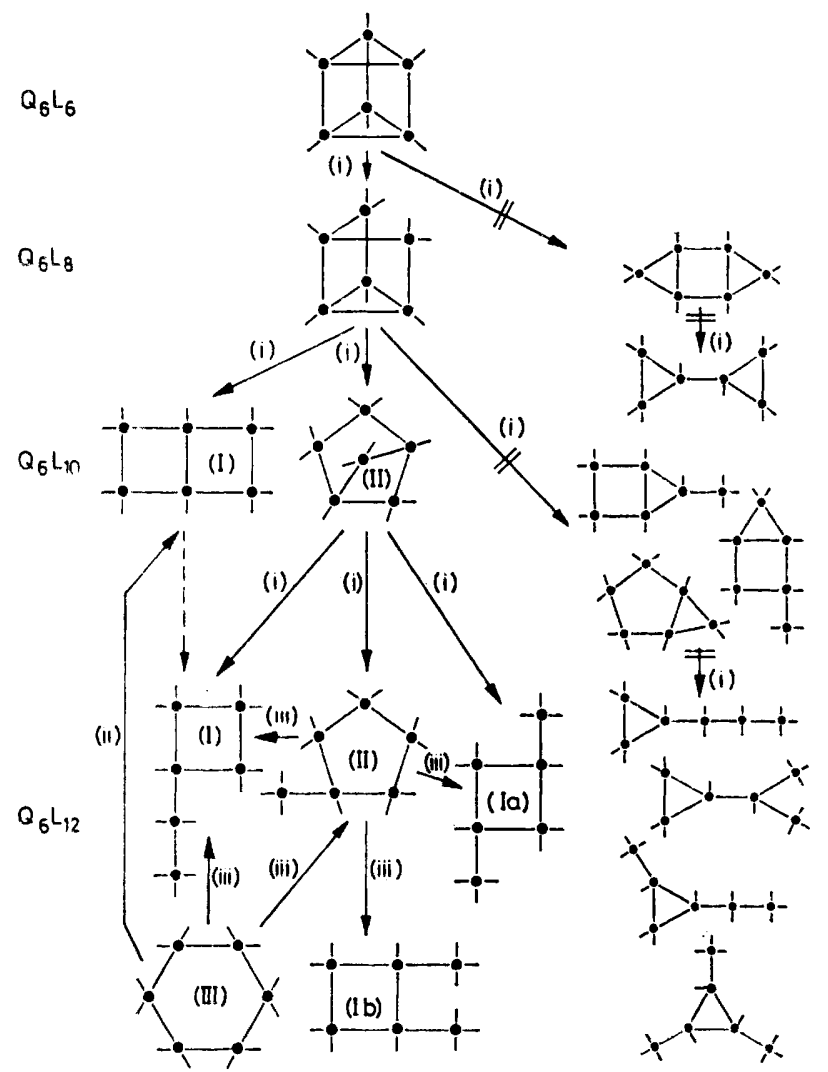

FIGURE 8 Chemical and structural transformations of $Q_{6} L_{6}$ and $Q_{8} L_{12}$ (III) induced by proton-donor catalysts. See text for description of reaction types (i), (ii), and (iii)

On the other hand, it is seen from Figure 3 that, regarding only the important reaction participants, both $Q_{6} \mathrm{~L}_{6}$ and $\mathrm{Q}_{6} \mathrm{~L}_{8}$ are represented by one, $\mathrm{Q}_{6} \mathrm{~L}_{10}$ by two, and $\mathrm{Q}_{6} \mathrm{~L}_{12}$ by three peaks in the chromatograms. These facts are sufficient for presenting the reaction pathway in the form of a most probable structural representation.

The entire pathway starting from $Q_{6} L_{6}$ and resulting in $Q_{6} \mathrm{~L}_{12}$, which may be realised in principle by type (i) reactions, is shown in Figure 8. It is evident that when type (ii) reactions are considered, the same pathway will apply in the opposite direction. Encountering also type (iii) reactions, the number of the possible geometric isomers would increase further and the scheme of the reaction pathway becomes rather confusing. Therefore type (ii) and type (iii) reactions are shown only in those cases when their existence is experimentally proved.

The occurrence of all transformations given on the lefthand side of Figure $\mathbf{8}$ follows from the experimental results discussed; there is no evidence for the participation in the transformations of the structures shown on the right-hand side of Figure 8. The comparatively high difference between the number of the possible and realised transformations suggests certain preferences of definite reaction routes.

In order to follow the pathway of type (i) reactions in Figure 8 , one can start from the fact that the structures of $\mathrm{Q}_{6} \mathrm{~L}_{6}, \mathrm{Q}_{6} \mathrm{~L}_{10}$ (I), and $\mathrm{Q}_{6} \mathrm{~L}_{10}$ (II) are known from previous work. ${ }^{7,11}$ These structures predetermine the structure of the transitional $Q_{6} \mathrm{~L}_{8}$ species, provided that only one-step type (i) reactions are to be considered both in the production and consumption of $\mathrm{Q}_{6} \mathrm{~L}_{8}$.

Curves in Figure 4 furnish evidence for the stability of $Q_{6} L_{10}(I)$ and for the intermediate character of $Q_{6} L_{10}$ (II). Therefore, in the third reaction sequence mainly $Q_{6} L_{10}$ (II) is transformed into $Q_{6} L_{12}$ by one-step type (i) reactions.

Of the three possible reaction routes starting from $Q_{6} L_{10}$ (II) in Figure 8, mainly one, leading to the isomer $\mathrm{Q}_{6} \mathrm{~L}_{12}$ (II), is operative and this product is further converted into $Q_{6} \mathrm{~L}_{12}(\mathrm{I})$, as can be seen in Figures 4 and 6 . In order to select the proper structure for the intermediate $Q_{6} L_{12}$ (II), one can rely on its analogous behaviour to the $Q_{6} L_{10}$ (II) structure. Hence it is assumed that the cyclopentasilicate structure is the most probable one for $Q_{6} L_{12}$ (II).

Disregarding the cyclohexasilicate structure, three cyclotetrasilicate structures remain for the most stable $\mathrm{Q}_{6} \mathrm{~L}_{12}$ (I) isomer, i.e. for the product which seems to be the preferred one in the present reaction route $(c f$. Figures 4 and 6). It is evident from the chromatograms in Figure 3 that $Q_{6} \mathrm{~L}_{12}(\mathrm{I})$ is actually represented by two peaks (peak nos. 6 and 7 ). (Most probably all the three peaks would be observed on improving the separation conditions in the g.c. column.) The existence and importance of the type (ii) reaction in certain cases follows from Figure 6 . This type of reaction is demonstrated therefore in Figure 8, starting from the silylation product of dioptase, $\mathrm{Q}_{6} \mathrm{~L}_{12}$ (III).

Two arguments may be given for the cyclohexasilicate structure of this trimethylsilyl ester. (i) Several observations speak for the maintenance of the original silicate structure ${ }^{8}$ when trimethylsilylation is performed either by bis(trimethylsilyl)acetamide or by the Tamás method, but without Amberlyst treatment.,7 (ii) Taking into account the usual elution behaviour of members of certain homologous series in g.c., the retention value of the $Q_{6} \mathrm{~L}_{12}$ (III) peak points also to the cyclohexasilicate structure. ${ }^{11,14}$ Additional information is offered by the curves in Figure 6: slow type (i) reactions starting from $Q_{6} L_{10}(I)$ have also been found (dotted-line 
arrow in Figure 8). . These reactions also contribute to the formation of $\mathrm{Q}_{6} \mathrm{~L}_{12}(\mathrm{I})$ isomers.

The structural scheme outlined above suggests the first conclusion: all reactions occurring in the course of Amberlyst treatment tend to transform cyclic and polycyclic trimethylsilyl silicate anion structures, con-
The data in the Table demonstrate that the experiments reported here were performed under reaction conditions similar to those used in previous investigations ${ }^{3,5}$ in the course of Amberlyst-treatment following the trimethylsilylation of silicate species. Experimental details and a discussion on the particular effects of the

Comparison of conditions of Amberlyst treatments

\begin{tabular}{|c|c|c|c|c|c|}
\hline Compound & $\begin{array}{l}\text { Skeletal } \\
\mathrm{SiO}_{2} / \mathrm{mg}^{a}\end{array}$ & $\begin{array}{c}\text { Hexamethyldisiloxane/ } \\
\mu l\end{array}$ & $\begin{array}{l}\text { Diluent/ } \\
\mu l\end{array}$ & $\begin{array}{c}\text { Amberlyst/ } \\
\text { mg }\end{array}$ & Ref. \\
\hline $\begin{array}{l}Q_{6} L_{6} \\
Q_{6} L_{12} \\
\mathscr{Q}_{6} L_{12} \\
Q_{6} L_{12}\end{array}$ & $\begin{array}{l}\text { ca. } 0.3 \\
\text { ca. } 0.3^{3} \\
\text { ca. } 0.4^{b} \\
\text { ca. } 0^{b} 5^{b}\end{array}$ & $\begin{array}{r}200 \\
1000 \\
800 \\
900\end{array}$ & 200 & $\begin{array}{r}40 \\
500 \\
200 \\
200\end{array}$ & $\begin{array}{c}\text { This work } \\
\text { This work } \\
\mathbf{5} \\
\mathbf{3}\end{array}$ \\
\hline
\end{tabular}

taining cyclotrisiloxane, cyclopentasiloxane, and cyclohexasiloxane units, into structures containing only cyclotetrasiloxane units.

The absence of the structures shown in the right-hand side of Figure 8 underlines the main tendency of the transformations, namely the preferred cleavage of $\equiv \mathrm{Si}-\mathrm{O}-\mathrm{Si} \equiv$ bonds in cyclotrisiloxane structural units. This preference follows from the strained bond system of the trimeric siloxane ring. The structures made up of cyclotetrasiloxane units or cyclotetrasiloxane chain combinations seem to be especially stable and represent therefore the end-products of the transformations discussed.

Further it can be concluded that chemical reactions of the trimethylsilyl silicates catalyzed by Amberlyst 15 play an important role in side reactions observed in the course of the trimethylsilylation procedures including after-treatment with Amberlyst. ${ }^{1-3,5}$ Hexamethyldisiloxane, the usual solvent in trimethylsilylation reactions, always takes part in the side reactions when $\equiv \mathrm{Si}-\mathrm{O}-\mathrm{Si} \equiv$ bond cleavage may occur upon proton catalysis.

The Role of Different Reaction Conditions in the Transformations of Trimethyl Silicates induced by Protondonor Catalysts.- The experiments and their interpretation outlined above suggest the decisive influence of several experimental factors on the nature and rate of the transformations discussed. silicate concentration, proton concentration, the nature of the proton source and that of the solvent will be published separately.

We thank Dr. J. Tamás and Dr. K. Ujszászi, Hungarian Academy of Sciences, Central Institute for Chemistry, for the determination of the elementary compositions of $Q_{6} L_{8}$ and $Q_{6} \mathrm{~L}_{12}$ isomers with the help of mass spectrometry.

[9/1960 Reccived, 10th December, 1979]

REFERENCES

1 C. W. Lentz, Inorg. Chem., 1964, 8, 574.

2 J. Götz and C. R. Masson, J. Chem. Soc. $(A), \mathbf{1 9 7 0 ,} 2683$

3 H. P. Calhoun and C. R. Masson, J.C.S. Dalton, 1978, 1342

4 G. Garzó, D. Hoebbel, Z. J. Ecsery, and K. Ujszászi, $J$. Chromatography, 1978, 167, 321.

${ }^{5}$ F. D. Tamás, A. K. Sarkar, and D. M. Roy, Hung. J. Ind. Chem., 1977, 5, 115 .

G H. P. Calhoun and C. R. Masson, J.C.S. Chem. Comm., 1978,560 .

7 D. Hoebbel, G. Garzó, G. Engelhardt, R. Ebert, E. Lippmaa, and M. Alla, $Z$. anorg. Chem., in the press.

8 H. G. Heide, K. Boll-Dornberger, E. Thilo, and E. M. Thilo, Acta Cryst., 1955, 8, 425.

9 J. Tamás and K. Ujszászi, unpublished work.

10 N. G. Polyansky, 'Catalysis with Ion-exchangers,' Isd. Khimiya, Moscow, 1973, pp. 15-26.

${ }_{11}$ D. Hoebbel, G. Garzó, G. Engelhardt, H. Jancke, P. Franke, and $W$. Wieker, $Z$. anorg. Chem., 1976, 424, 115 .

12 W. Noll, 'Chemie und Technologie der Silicone,' Verlag Chemic, Weinheim, 1960, pp. 139-141.

${ }_{13}$ G. Garzó and G. Alexander, Chromatographia, 1974, 7, 190.

14 G. Garzó and G. Alexander, Chromatographia, 1971, 4, 554. 